\title{
Influence of Crystal Orientation on the Freckle Formation in Directionally Solidified Superalloys
}

\author{
Dexin $\mathrm{Ma}^{1, \mathrm{a}}$, Michael Mathes ${ }^{2, \mathrm{~b}}$, Bin Zhou ${ }^{3, \mathrm{c}}$, Andreas Bührig-Polaczek ${ }^{1, \mathrm{~d}}$ \\ ${ }^{1}$ Foundry Institute, RWTH University Aachen, Germany \\ ${ }^{2}$ ACCESS Institute, RWTH University Aachen, Germany \\ ${ }^{3}$ Departmemt MTM, Katholieke Universiteit Leuven, Belgium \\ ad.ma@gi.rwth-aachen.de, ${ }^{\mathrm{b}} \mathrm{m}$.mathes@access.rwth-aachen.de, \\ 'bin.zhou@mtm.kuleuven.be, dsekretariat@gi.rwth-aachen.de
}

Key words: Superalloy, single crystal, freckles, crystal orientation

\begin{abstract}
Freckle occurrence is known to be dependent on the casting size and the superalloy components with large cross section are more prone to freckle formation. In our directional solidification experiment of superalloys freckles are observed in some thin samples, while some significantly thicker samples in the same shell mould cluster remain freckle free. The EBSD analysis shows that the samples with freckles have a good $<001>$ axial orientation, while in freckle free samples the primary dendrite stems diverge from the sample axis. In an analogue system the flow behaviour through a mushy zone was simulated, in which the dendrite network was placed in different orientation. The $<001>$ dendrite array shows the lowest resistance to the vertical melt flow. As the dendrite alignment diverges from $<001>$ orientation, the flow speed slows down and reaches the minimum at the $\langle 111>$ orientation. This orientation effect of the interdendritic permeability explains well why the crystal orientation plays such an important role in the occurrence of freckles in superalloy castings.
\end{abstract}

\section{Introduction}

Freckles are presently one of the main cast defects in the single crystal components made of superalloys. Freckles are observed as long and narrow trails, aligned roughly parallel to the direction of gravity. It is now generally agreed that freckles are the product of specific fluid flow patterns, known as thermosolutal convection, originating in the interdendritic liquid during solidification [1]. This flow is driven by a density inversion occurring in the mushy zone as a result of interdendritic segregation. With solidification oriented anti parallel to the direction of gravity, the compositional variation associated with solute segregation in the dendritic mushy zone results in a density imbalance between the bulk and the segregated liquid.

Freckle occurrence is known to be dependent on three factors: alloy chemistry, casting conditions and casting size. For the defined alloy system and casting process, the components with large cross section are more freckle prone. In contrast, freckles are normally not present in small diameter rods. In blade casting freckles are usually found in the root rather than in the airfoil section. This represents a critical diameter $\mathrm{D}_{0}$ or a minimum area $\mathrm{A}_{0}$, in order to support the fluid flow pattern associated with freckle formation. In the experiments of Giamei and Kear with superalloy Udimet700 [2], the minimum area was found to be $\mathrm{A}_{0} \approx 322 \mathrm{~mm}^{2}$. The corresponding diameter $\mathrm{D}_{0}$ is then about $20 \mathrm{~mm}$. The number of freckle lines $(\mathrm{N})$ increases with increasing bar diameter. It was found that $\mathrm{N}$ is proportional to the cross section area $\mathrm{A}$, and the freckling density is proportional to the local diameter. It is also referred to as the size effect of the freckle formation.

In our recent directional solidification (DS) experiments with superalloys, however, it was found that the bigger diameter specimens are not always more prone to freckles than the smaller ones. Thus the size effect on the freckle formation cannot be convincingly confirmed. The aim of the present work is to explain this phenomenon and to detect whether there are other factors influencing the freckle formation. 


\section{Directional solidification experimental and specimen examination}

The single crystal (SC) casting experiments were carried out using the conventional Bridgman technique in a vacuum environment. CMSX-4 was chosen for the investigation since it is a typical freckle prone superalloy [3]. Several cylindrical bars with constant or varied diameter were used to verify the influence of the casting size on the freckles formation (Fig. 1). The shell mould clusters with the components assembled around a central rod was mounted in the Bridgman furnace. After preheating and pouring the superalloy melt, the shell mould was withdrawn out of the heating zone through the baffle into the cooling zone. The selector method was used for the single crystal solidification of the components. Heater and pouring temperature of $1500{ }^{\circ} \mathrm{C}$ and withdrawal velocity of $1 \mathrm{~mm} / \mathrm{min}$ were applied. Macroetching was employed to determine the freckle chains on the surface of the directionally solidified specimens.

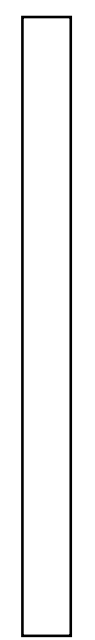

Diameter: 12

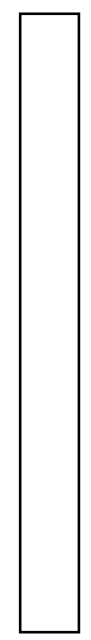

15

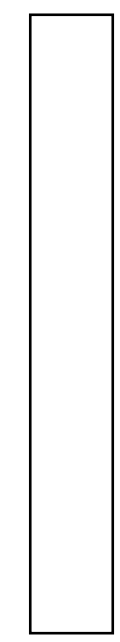

$20 \quad 25$

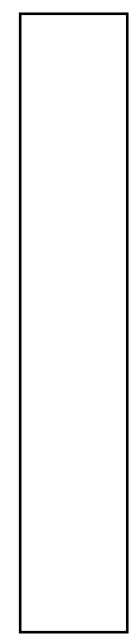

$12 / 15 / 20 / 25$

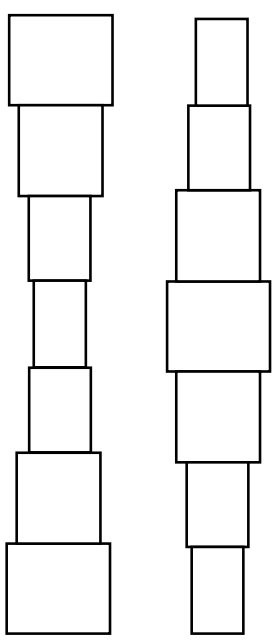

with constant or varying diameter. The entire length of the specimens is $175 \mathrm{~mm}$. The step length with varying diameter (the right 2 geometries) is $25 \mathrm{~mm}$.

During structure investigation it was found that the freckles, if any, are only found on the surface of the side facing the central rod. On the side facing the heater, no freckles were observed. This indicates an unsymmetrical thermal condition between the inner and outer side of the casting cluster. The observed freckles were normally formed at the top parts of the specimens rather than at the bottom part, revealing that the solidification condition became relatively worse during the upwards solidification process.

The size effect on the freckle formation cannot be generally confirmed. The observation is not consistent with the belief that large components are significantly more prone to freckling than smaller ones. In some thinner specimens freckles occurred while some thicker ones remained freckle free. With careful observation of the surface of the specimens it can be found that in the specimens with freckles the dendrites are aligned roughly parallel to the long axis of the specimens, while in the specimens without freckles the dendrite alignment is inclined. Since the alignment of the dendrites is defined by the crystal orientation, it seems that the freckle formation is strongly influenced by the crystal orientation of the specimens.

The orientations in the specimens were investigated using EBSD (Electron Backscatter Diffraction). Prior to analysis, the transverse sections were taken perpendicular to the long axis of the specimens (the heat flux direction). The samples were sequentially ground with silicon carbide paper of different grit sizes. Subsequently the samples were polished with diamond paste on a polishing wheel carrying standard cotton cloth. The polished samples were then examined by means of EBSD analysis. Fig. 2 shows the measured pole figures on the sections of some cylindrical specimens of different diameters. 


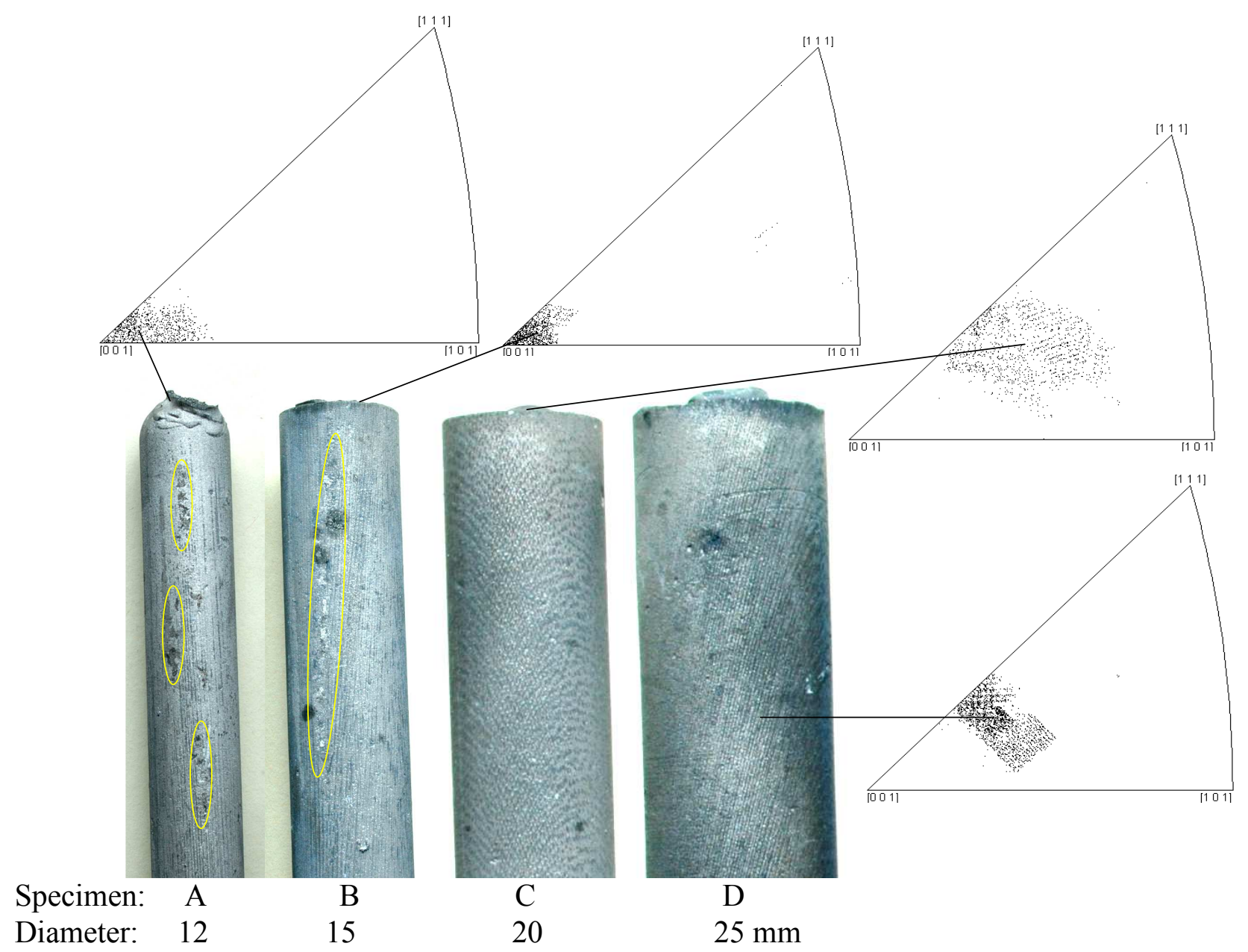

Fig. 2: Photographs of SC specimens with different diameters and pole figures exhibiting their crystal orientations. The circles indicate the freckles, which occur only on the well aligned specimens (A and B), in spite of their smaller diameters.

In the specimens with well aligned dendrites, as expected, the $<100>$-orientation is nearly identical to the specimen axis and hence to the solidification direction. In this case the specimens are very prone to freckle formation in spite of their small diameter (specimen A and B in Fig. 2). On the other hand, in the specimens with bad aligned dendrites, no freckles have been observed, although their diameter is significantly increased (specimen C and D in Fig. 2). It is interesting to note that the freckles could occur in the thinnest specimen A with a diameter of only $12 \mathrm{~mm}$, but not in the thickest specimen D with a diameter of $25 \mathrm{~mm}$, although the cross section area becomes more than four times larger. It should be pointed out that all specimens were directionally solidified in the same shell mould cluster and hence under the same process condition. The only difference is that the dendrite alignment in the thicker specimen $\mathrm{D}$ deviates about 18 degrees from the specimen axe, while in specimen A the average deviation is only about 6 degrees with respect to gravity. From the above observation, the well known size effect, namely larger sizes are favourable to freckle formation, cannot be recognized.

The specimens with a stepwise expansion of the cross-section show the same tendency (Fig. 3). In the Specimen E the dendrite alignment in $<100>$-orientation is nearly identical to the specimen axe and freckles could be observed in each stage of the specimen. When increasing the diameter from 15 over 20 to $25 \mathrm{~mm}$, the freckle formation becomes more and more pronounced. Only in this case, the well known size effect of the component on the freckle formation could be confirmed. In the specimen $\mathrm{F}$, the dendrite alignment deviates far from the solidification direction. As a result, no freckles were found on the surface of this specimen, even at the top stage with the maximum diameter of $25 \mathrm{~mm}$. This indicates once again that the freckle formation is noticeably more sensitive to dendrite orientation than to the diameter of the components. 


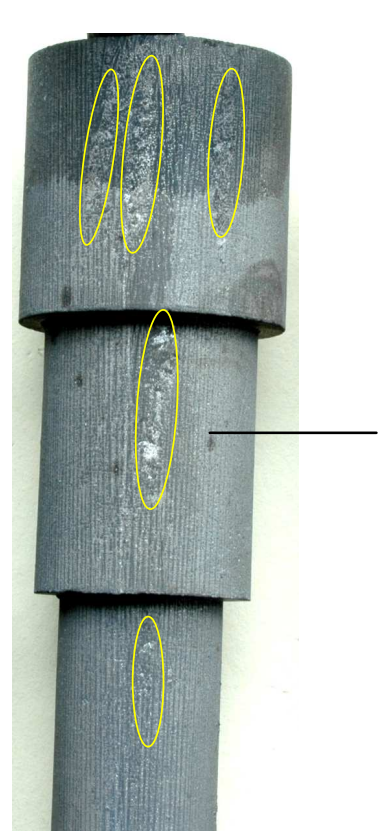

E

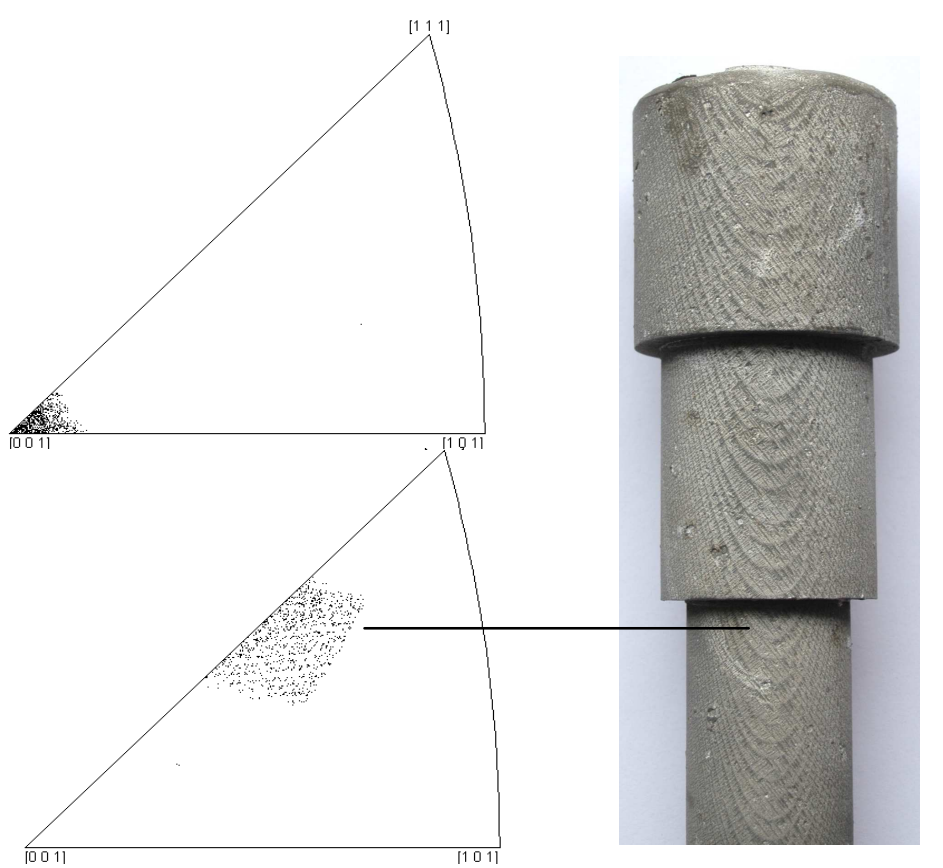

$\mathrm{F}$

Fig. 3: Photographs of SC specimens with stepwise varied diameter (15, 20 and $25 \mathrm{~mm})$. The pole figures indicate their crystal orientations. Freckles occur in specimen E, as indicated by circles, while specimen F remains freckle free.

It is well known that the freckles are the product of thermosolutal convection, originating in the interdendritic liquid during solidification and following the direction of the driving force caused by the density inversion. From the observation in Fig. 2 and $\mathbf{3}$ it can be assumed that the dendrite alignment may influence the interdendritic fluid flow and hence the propensity to form freckles. This will be investigated further by the following simulation.

\section{Computer simulations with MAGMASOFT}

In the present work a computer simulations was carried out to simulate the flow behavior in mushy zone. The simulation was run in MAGMASOFT with the form-filling module. The mushy zone was represented by the core in this module, which shall remain solid during the form filling, and has the same geometry as the dendrite networks. As shown in Fig. 4a, the used columnar dendrite consists of a trunk of $2 \mathrm{~mm}$ in diameter and the secondary arms whose diameter, length and spacing are designed to be $1.5 \mathrm{~mm}$. These dendrites were placed in different orientations in the casting cavity. As reference the columnar dendrite array was firstly aligned vertically, to exhibit the $<100>$ orientation of the dendrites in the long axis of the casting (Fig. $\mathbf{4 b}$ ), anti parallel to the gravity direction. The other orientations, $<110\rangle$ and $\langle 111\rangle$, can be also realized by inclining the dendrite arrays in defined directions to defined degrees (Fig. $4 \mathbf{c}$ and $\mathbf{d}$ ). 


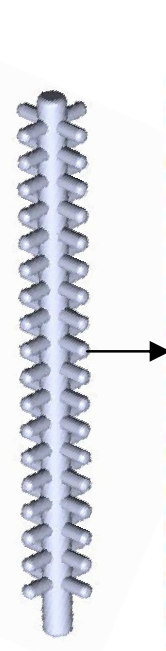

(a)

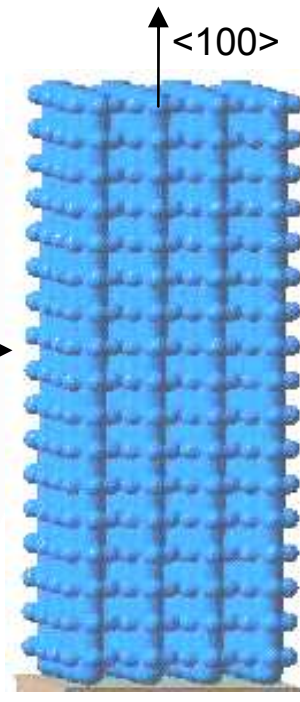

(b)

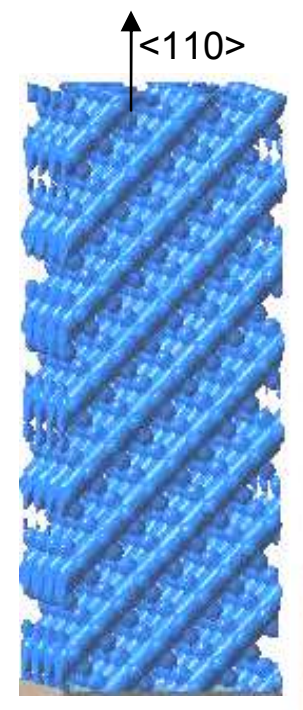

(c)

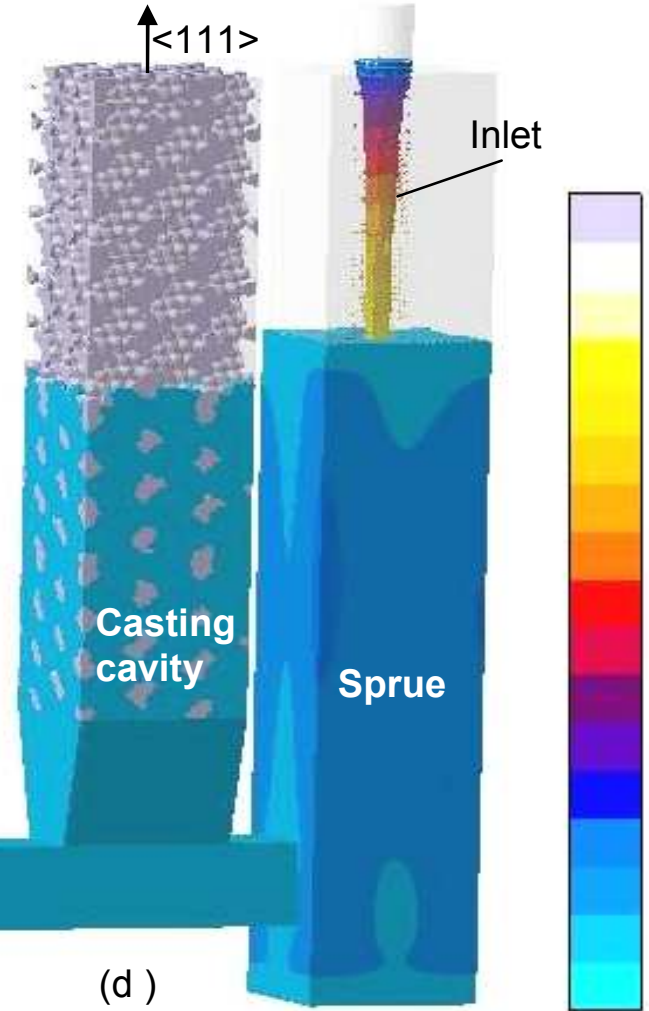

(d)
Velocity

$[\mathrm{cm} / \mathrm{s}$ ]

Empty

113.8

105.7

97.6

89.4

81.3

73.2

65.0

56.9

48.8

40.7

32.5

24.4

16.3

8.1

0.0 simulation of filling process (d).

The melt was fed into the casting system to go through the core geometry, similar to the flow in the mushy zone (Fig. 4d). The simulation was based on the mould-filling module for the sand casting, which was well established during the earlier practices [4]. After the entrance of the melt into the casting cavity a stable flow can soon be established. Fig. 5 shows the simulated melt flow state within the investigated dendrite networks at the same filing time ( 10 seconds after pouring).

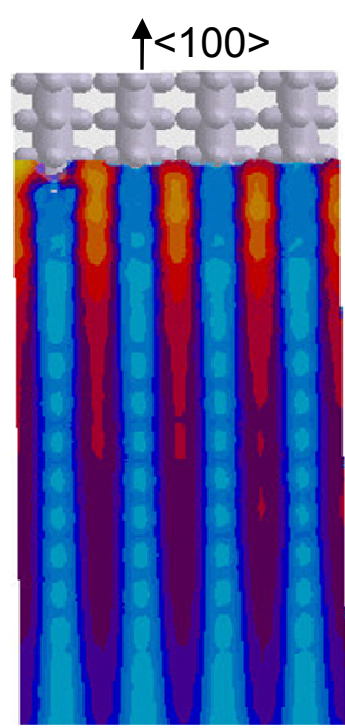

(a)

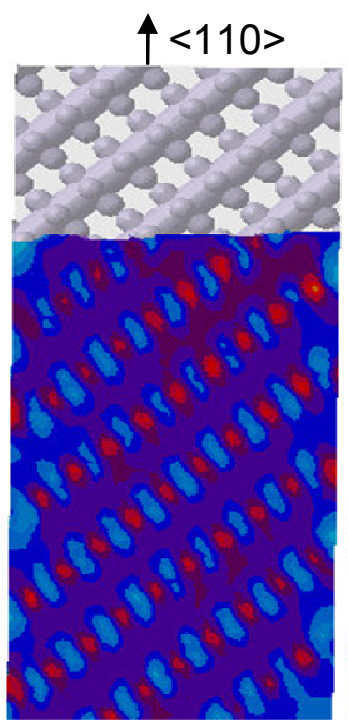

(b)

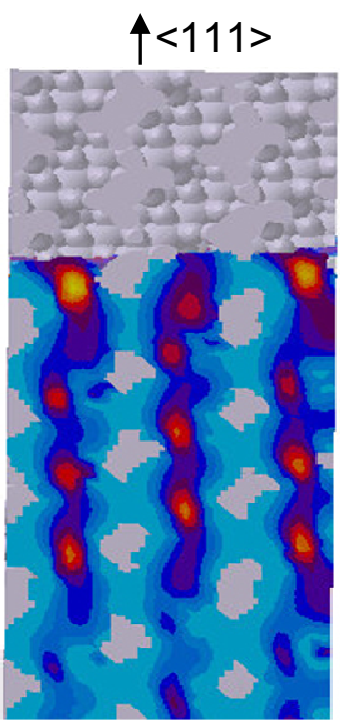

(c)

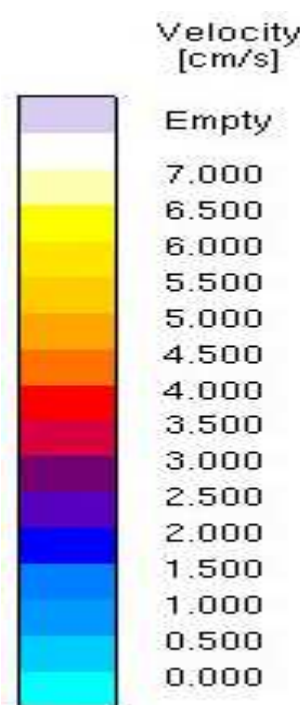

Fig. 5: Flow behavior comparison among $<100>,<110>$ and $<111>$ orientation dendrite array. After the same filling time ( 10 seconds), the flow front in the $<100>$ orientation reached a clearly higher level than that in the $<110>$ and $<111>$ orientation. 
In Fig. 5 the relationship between the height of the melt flow front and the dendrite orientations is well demonstrated. As can be seen, the upward flow front in the dendrite array with $<100>$ orientation advanced the most (Fig. 5a). In the dendrite arrays with $<110>$ and $<111>$ orientation the melt flowed significantly slower (Fig. $5 \mathbf{b}$ and $\mathbf{c}$ ).

In the above form-filling simulation, all conditions for the melt flow (pouring pressure, fraction solid in mushy zone and dendrite spacing) are the same, except that the orientations of the dendrites were varied. Therefore the dendrite orientation is the only reason for the difference in flow behavior. It is reasonable to assume that the melt flows more easily along the channels among the primary dendrite trunks than in any other directions. For the vertical dendrite array, the driving force and the primary dendrite trunks are identical, thus enabling the fast melt flow. When the dendrites are inclined to a defined angle, the vertical melt flow must pass through the dendrite trunks with the same angle. Increasing the inclination angle, more dendrite trunks have to be passed through, leading to the increase in the resistance to the upward flow. As well known, freckles are believed to be generated as a result of thermalsolutal convection in upwards direction against the dendrite resistance. Thus the well orientated single crystal castings are more freckle-prone because the vertical aligned dendrite arrays have the best permeability and are then favorable for the onset of the vertical thermosolutal convection. In contrast, the inclination of the dendrites becomes a stabilizing factor for the interdendritic liquid to suppress the vertical thermosolutal convection and the subsequent formation of freckle defects.

Auburtin et al. [5] studied the influence of solidification front angle on freckle formation by tilting the Bridgman-furnace used for DS experiments. It was fund that tilted castings exhibited freckles, whereas vertical castings were freckle-free. In the present work all SC-specimens were vertically solidified and the influence of the crystal orientation of the specimens on the freckle formation has been detected. This could add new insight towards understanding the freckle arising mechanisms. Furthermore, the dependence of freckling on crystal orientation should be taken into account in the modelling and simulation to provide more correct prediction of freckle formation in superalloy castings.

\section{Summary}

Directional solidification experiments of superalloy CMSX-4 have been performed to investigate the freckle formation. The freckles are observed in some small diameter samples while some significantly bigger samples in the same shell mould cluster remain freckle free. The EBSD analysis shows that the samples with freckles have a good $<001>$ axial orientation, while in freckle free samples the $<001>$ primary dendrite trunks deviates clearly from the sample axis. It is then proved that the freckle formation is noticeably more sensitive to dendrite orientation than to the component diameter. In an analogue system the flow behaviour through a mushy zone was simulated, in which a dendrite network was placed in different orientation. The $<001>$ dendrite array shows the least resistance to the melt flow. With increasing the deviation of the dendrite orientation the flow speed slows down and reaches the minimum at the $<111>$ orientation. Due to the increased resistance to the vertical thermosolutal convection, the inclination of the dendrite arrays becomes a stabilizing factor to suppress the vertical thermosolutal convection and the subsequent formation of freckle defects. Therefore the orientation effect of the interdendritic permeability well explains why the samples with $<001>$ axial orientation are most freckle prone.

\section{References}

[1] S.M.Copley, A.F.Giamei, S.M.Johnson, M.F.Hornbecker: Metall. Trans., 1 (1970) p. 2193

[2] A.F.Giamei, B.H.Kear: Metall. Trans., 1 (1970) p.2185

[3] R.Schadt, I.Wagner, J.Preuhs, P.R.Sahm: Superalloy 2000, Edited by T.M.Pollock et al, TMS, p. 221

[4] T.Midea: Foundry Practice, 241 (2004) p.15

[5] P.Auburtin, T.Wang, S.L.Cockcroft, A.Mitchell: Met. Mat. Trans. B, 31B (2000) p. 801 